\title{
Pheochromocytoma and pregnancy with abruptio placenta
}

\author{
Noureddine Korichi, Nissar Shaikh*, Gisha Mathew, \\ Mahommad Amin Alloub, Ibrahim Boursaly, Nick Scott
}

Department of Anaesthesia/ICU and Perioperative Medicine, Weill Cornell Medical College, Doha, Qatar, Dubai

Received: 3 June 2014

Accepted: 1 July 2014

*Correspondence:

Dr. Nissar Shaikh,

E-mail: nissatfirdous99@gmail.com

(C) 2014 Korichi $\mathrm{N}$ et al. This is an open-access article distributed under the terms of the Creative Commons Attribution Non-Commercial License, which permits unrestricted non-commercial use, distribution, and reproduction in any medium, provided the original work is properly cited.

\begin{abstract}
A 41 year old previously healthy woman (Gravida 4, para 3) was presented to our hospital at 29 weeks gestation, with bleeding Per Vagina (PV) and severe hypertension (190/100). She underwent a routine obstetric examination at 12 weeks gestation and since then she has not undergone any antenatal follow-up. She developed episodes of severe headache, dizziness, sweating, and nausea. She visited a private hospital and was noted to be severely hypertensive $(190 / 120)$ with headache and palpitations. An ultrasound abdomen was done which showed left suprarenal mass, and a diagnosis of pheochromocytoma was made. She was treated there with antihypertensive medications. When Blood pressure got controlled, she was discharged against medical advice. At 29 weeks, she suddenly developed severe headache and bleeding PV. She visited our centre and was diagnosed to have abruptio- placenta with foetal distress. An emergency caesarean section was done, and following which the patient was treated in the ICU with antihypertensive under invasive monitoring. An MRI demonstrated a left pheochromocytoma. A laparoscopic adrenelectomy was planned later and she got discharged on antihypertensive following an uneventful period of recovery. She got operated later in her country. A laparoscopic left adrenelectomy was done. She is off all medications now and is currently asymptomatic.
\end{abstract}

Keywords: Pheochromocytoma, Abruptio placenta, Lower section caesarean section

\section{INTRODUCTION}

A pheochromocytoma in a pregnant patient is one of the most threatening medical conditions for the mother, foetus and the physician. Although extraordinarily rare with a frequency of $0.002 \%$ of all pregnancies, the tumour is notorious for its devastating consequences. As in non-pregnant patients, the signs and symptoms are quite variable but not specific with hypertension being one of the most prominent signs. Confusion with the much more prevalent forms of pregnancy related hypertension is the main cause of overlooking the diagnosis. If undiagnosed, maternal and foetal mortality is around $50 \% .^{1}$ The transient excessive maternal levels of catacholamines in patients with pheochromocytoma may have deleterious effects on uteroplacental circulation. Extreme vasoconstriction of this specific and vulnerable vascular bed may be responsible for the occurrence of placental abruption, intrauterine hypoxia and IUGR, thus imposing a serious risk to the foetus. We report case of placenta previa with pheochromocytoma.

\section{CASE REPORT}

A 41 year old previously healthy woman (Gravida 4, para 3), presented to our hospital at 29 weeks gestation with bleeding per vagina and severe hypertension. She was conscious and oriented. She underwent a routine obstetric examination at 12 weeks gestation and since then she has not undergone any further examination until 24 weeks. She developed episodes of severe headache, dizziness, sweating and nausea at 24 weeks. She visited a private hospital and was noted to be severely hypertensive (190/120). Routine biochemical investigations showed 
normal values except for a high random blood sugar of $158 \mathrm{mg} / \mathrm{dl}$. The renal function test, liver function test, Uric acid, Serum albumin all found to be normal. She did not have proteinuria. An ultrasound abdomen was done which showed a $0.6 / 4.5 \mathrm{~cm}$ left supra renal mass. 24 hour urinary vanillylmandelic acid was found to be elevated (69 micromols/day). A diagnosis of pheochromocytoma was made. She was treated in the ICU initially with labetalol infusion then with tab nifedipine, tab methyldopa $500 \mathrm{mg}$, and Tab Aspirin 75mg OD. When the Blood pressure got controlled she got discharged against medical advice. Since then she continued the antihypertensives at home and was having episodes of headache, nausea and palpitations in between. At 29 weeks she suddenly developed bleeding Per Vagina and she was brought to our hospital. She had severe hypertension with a BP of 190/120 and palpitations (HR 106/mt). Per abdominal examination showed a uterus of 30 weeks size, with normal uterine tone and fetal heart sound positive. A diagnosis of abuptio placenta was made. She was a non-smoker and non-drinker and had no reported allergies. She had three prior uncomplicated pregnancies with term vaginal deliveries. Family history was negative for MEN syndromes and pheochromocytoma. Her physical examination was otherwise unremarkable. She received tab nifedipine 60 $\mathrm{mg}$ followed by tab labetalol $200 \mathrm{mg}$ in the emergency. An IV access was obtained with an $18 \mathrm{G}$ cannula and blood was sent for routine biochemical investigations. The patient was then immediately shifted to the OR for an emergency caesarean section. In the OR, a quick general physical examination was done and the routine monitors like NIBP, $\mathrm{SPO}_{2}$ and ECG were connected. A Foleys catheter was also inserted. She had her last meals 2 hours ago. $30 \mathrm{ml}$ of $0.3 \%$ sodium citrate was given along with IV metoclorpromide $10 \mathrm{mg}$. Her body weight was $100 \mathrm{~kg}$. Her BP was $180 / 110$ and PR was $96 / \mathrm{mt}$. The chest was clear and heart sounds were normal. The patient was positioned with left uterine displacement. After pre oxygenating for 3 minutes, a crash induction was done with lidocaine $1 \mathrm{mg} / \mathrm{kg}$, propofol $2 \mathrm{mg} / \mathrm{kg}$ and scoline $1.5 \mathrm{mg} / \mathrm{kg}$. Anaesthesia was deepened with sevoflurane prior to laryngoscopy. Cricoid pressure was given and she was intubated with 7 sized ET tube and after confirmation of the correct tube placement, she was connected to the ventilator. She received injection cisatracurium $0.1 \mathrm{mg} / \mathrm{kg}$ and was maintained on airoxygen mixture of $0.5 \%$ each and sevoflurane $0.5-1 \%$. An orogastric tube was inserted and the contents were aspirated. After the delivery of the baby injection fentanyl $200 \mathrm{mcg}$, and injection syntocinone $10 \mathrm{U}$ iv was given. Another $20 \mathrm{U}$ syntocinone was added to the drip. Following induction the blood pressure dropped to100/60 which was picked up by giving $500 \mathrm{ml}$ Ringer Lactate as a fast IV drip. Injection phenylephrine 50 micrograms per $\mathrm{ml}$ and injection labetalol was prepared and kept. In addition to the 18 Gauge intravenous cannula which was already present, another IV access was obtained with a 16 gauge cannula and crystalloids were started. Colloids were withheld for fear of pulmonary oedema. Blood was sent for cross matching. Since the uterus was relaxed, an additional 10 Units syntocinone was added to the drip. The baby's APGAR was 3/5. He was resuscitated and intubated and shifted to the (neonatal intensive care unit) NICU. At the end of the procedure, she received full analgesia with a total of $10 \mathrm{mg}$ of morphine. The patient was reversed with neostigmine $2.5 \mathrm{mg}$ and glycopyrrolate $0.4 \mathrm{mg}$ and extubated. She also received inj. dexamethasone $8 \mathrm{mg}$ iv, inj. ondansetrone $4 \mathrm{mg}$ iv, inj. paracetamol $1 \mathrm{gm}$ iv. The procedure took one hour, the blood loss was $900 \mathrm{ml}$ and the urine output was $300 \mathrm{ml}$. She received a total of $1400 \mathrm{ml}$ of crystalloids. Her $\mathrm{Hb}$ report came out to be $12.8 \mathrm{gm} / \mathrm{dl}$, so blood was not asked for. Immediately after extubation, the spo 2 showed $85 \%$. On auscultating the chest, there were few basal crepitations. Inj. Lasix $20 \mathrm{mg}$ iv was given. The $\mathrm{SPO}_{2}$ improved to $98 \%$ gradually. The $\mathrm{BP}$ was stable throughout the procedure and was maintained by incremental injections of propofol 20-30 mg each and did not require any antihypertensive medication. In the end, the patient was fully conscious with a BP of $150 / 90$ and a normal SPO2 of $98 \%$. She was shifted to the ICU for monitoring and control of $\mathrm{BP}$ where a radial artery cannulation was done and BP monitored. In the ICU, the BP rose to $180 / 100$ and labetalol infusion was started. Postoperative pain was controlled with patient controlled analgesia using pethidine. The reduction in intraabdominal pressure and presumed decrease in mechanical stress on the adrenal tumour following delivery of the foetus did not lead to a reduction in the antihypertensive drug requirement post operatively. She was shifted to the ward after 72 hours. She was further treated with nifedipine 60 bid and Tab labetalol $400 \mathrm{mg}$. The post op period was uneventful. The patient was referred to the surgeon and the endocrinologist. A series of biochemical investigations were done postoperatively to confirm the diagnosis. An MRI demonstrated a left suprarenal mass of more than $5 \mathrm{~cm}$. A multidisciplinary conference involving endocrinology, anaesthesiology, general surgery and obstetrics was held to determine the most appropriate management for this patient. A laparoscopic adrenalectomy was planned but since she wanted to get operated in her own country, she got discharged. She was advised to continue tab nifedipine $40 \mathrm{mg}$ bid and $\mathrm{Tab}$ methyldopa $500 \mathrm{mg}$ bid. The baby was discharged home after a short period of treatment in the NICU. She got operated later in her country. A laparoscopic left adrenelectomy was done and subsequently it was proven histopathologically to be pheochromocytoma with $5.1 / 3.1 / 3 \mathrm{~cm}$ in size. The patient is off all medications now and is currently asymptomatic.

\section{DISCUSSION}

Pheochromocytoma is a catecholamine secreting tumour derived from chromaffin tissue. It occurs in one or both adrenal glands $(85 \%)$ or in the sympathetic ganglia $(15 \%) .{ }^{1}$ It is extraordinarily rare in pregnancy with a frequency of $0.002 \%$ but is notorious for its devastating consequences. ${ }^{2}$ Signs and symptoms include paroxysmal 
or sustained hypertension, headache, sweating, palpitations, nausea, tremor and anxiety. As in nonpregnant patients, the signs and symptoms in pregnancy are quite variable but not specific with hypertension being one of the most prominent signs. Since it is difficult to distinguish between pre-eclampsia and pheochromocytoma in pregnancy, it is important to maintain a high index of suspicion in patients with these symptoms. Symptoms can be worsened in pregnancy, because of the increased vascularity of the tumour and mechanical factors such as pressure from the enlarging uterus or foetal movements which can stimulate catecholamine secretion. ${ }^{3}$ While pre-eclampsia presents with protienuria and hypertension occurring after the $20^{\text {th }}$ week of gestation, pheochromocytoma is rarely associated with protienuria and causes hypertension in the entire pregnancy. ${ }^{4}$ An elevation of urinary and plasma catecholamines and their metabolites is the most sensitive and specific diagnostic finding. Of all the available compounds that can be measured, fractionated metanephrines (nor-metanephrine and metanephrine measured separately) are the preferred metabolites for the initial test. ${ }^{5}$ An initial test should have the strongest power to exclude the tumour as reliably as possible so that no tumour is missed. Metanephrines, measured either in the blood or in the urine, have this power since they have the highest sensitivity and highest negative predictive value. ${ }^{6}$ The transient excessive maternal levels of catecholamine's had deleterious effects on uteroplacental circulation. Extreme vasoconstriction of this specific and vulnerable vascular bed may be responsible for the occurrence of placental abruption, intra uterine hypoxia and IUGR.

Antenatal diagnosis is essential to improve maternal and foetal outcome. If undiagnosed, maternal and foetal is around $50 \%$. $^{7,8}$ While Harper et al. found an overall maternal mortality of $17 \%$ and foetal mortality of $26 \%$, with antenatal diagnosis, maternal mortality is reduced to nearly $0 \%$ and foetal loss to $15 \% .^{9-13}$ The primary goal in the management of pheochromocytoma in pregnancy are early diagnosis, avoidance of a hypertensive crisis during pregnancy and delivery and a definitive surgical treatment. A fatal hypertensive crisis can be precipitated by the mechanical effects of an enlarging uterus, uterine contraction or foetal movements, by vaginal delivery or general anaesthesia. ${ }^{9,14}$ This hypertensive crisis can lead to haemorrhage and infarction in the vital organs, congestive cardiac failure, cardiac dysrythmias and death.

After biochemical confirmation of the diagnosis, the tumour can be localized safely during pregnancy by ultrasonography and MRI. ${ }^{15}$ USG has a sensitivity of 8997\% for adrenal masses and MRI avoids all radiation and allows sectional imaging to allow visualization of extra adrenal tumours and is the imaging procedure of first choice. ${ }^{5,9,16}$ The diagnostic sensitivity of MRI is almost $90-100 \%$. $^{1,5,17}$

Surgical excision is the definitive treatment. ${ }^{2,4,15-18}$ Timing of excision will depend on the gestational age at which the diagnosis is made, foetal development and the success of the treatment of maternal symptoms. ${ }^{4,11,19,20}$ The tumour can be excised immediately if the diagnosis is confirmed prior to 24 weeks gestation. ${ }^{4,11,20}$ Depending on the clinical circumstances, pregnancy can be terminated or continued. Second trimester is the safest period to do the surgery during pregnancy because of the risk of spontaneous abortion in the first trimester. Since adequate surgical exposure is restricted by the enlarging uterus after 24 weeks, the pregnancy can be allowed to continue until foetal maturity is reached, under cover of adrenergic blockers. In those patients, the tumour can be removed simultaneously with caesarean section or separately after delivery. Patients who are pre-treated with alpha blockers had a lower maternal mortality than those who have no alpha adrenergic blockade. ${ }^{21}$ Establishment of alpha adrenergic blockade generally requires 10-14 days of treatment. A beta blocker is also indicated if there is persistent tachycardia or cardiac dysrhythmias and usually responds following 2-3 days of treatment. Elective caesarean section is the preferred mode of delivery as a fatal hypertensive crisis can be precipitated by the uterine contractions and the maternal expulsive efforts during vaginal delivery. ${ }^{4,11,16}$ While the maternal mortality is $19 \%$ in caesarean sections, it is as high as $31 \%$ in vaginal delivery. ${ }^{12}$

Epidural, general or combined anaesthetic techniques has been used successfully for caesarean section. ${ }^{22}$ General anaesthesia is the preferred mode of anaesthesia which can be combined with epidural anaesthesia to eliminate the sympathetic stimulations. The greatest hemodynamic instability is anticipated during intubation of the trachea, tumour manipulation and following tumour ligation. The goal of anaesthetic management is the avoidance of drugs or events that will result in the stimulation of sympathetic nervous system. As our patient presented with foetal distress and her NPO status was not adequate we did a rapid sequence induction with propofol and scoline. We preferred propofol to thiopentone to avoid the increase in heart rate with thiopentone. All iv induction agents are safe except for ketamine which can cause a sympathetic stimulation. Due to the emergent situation an epidural catheter could not be inserted. Intra operative monitoring should aim at the early detection of catecholamine induced cardiovascular changes and includes arterial BP monitoring and pulmonary arterial catheterization. Since the baby was delivered within 3 minutes and the whole procedure lasted less than 40 minutes an arterial catheterization was not done. The vitals remained stable throughout the procedure.

Laryngoscopy and tracheal intubation can trigger an acute hypertensive crisis by the abrupt release of catecholamines causing uteroplacental insufficiency and foetal hypoxia. ${ }^{4}$ To minimize this a surgical depth of anaesthesia should be achieved (1.3 MAC) prior to any attempt at laryngoscopy and intubation. ${ }^{23}$ We deepened the anaesthesia with sevoflurane prior to laryngoscopy. Administration of a bolus of IV lidocaine (1-2 mg/kg) 
prior to induction also can attenuate catecholamine induced responses including cardiac dysrhythmias. Fentanyl was used as an adjunct to anaesthesia. Non depolarizing muscle relaxants with vagolytic or histamine releasing properties are best avoided. Cisatracurium was used in our patient because it has no vagolytic or histamine releasing properties. ${ }^{24}$ It undergoes degradation in plasma at physiological $\mathrm{PH}$ and temperature by organ independent Hoffman elimination. Anaesthesia can be maintained by a volatile anaesthetic and an opioid. We used sevoflurane because it does not sensitize the myocardium to catecholamines and decreases SVR without increasing the heart rate. ${ }^{25}$ Isoflurane can also be used safely but rapid increase in Isoflurane concentration can lead to transient increase in heart rate, arterial BP and plasma levels of nor epinephrine. ${ }^{25}$ Desflurane is best avoided as it stimulates catecholamine release when administered rapidly at high concentration. Halothane is also not preferred as it sensitizes the myocardium to circulating catecholamines.

Magnesium sulphate has also been used successfully as intra operative infusion at therapeutic levels. ${ }^{26}$ It inhibits the release of catecholamines from both the adrenal medulla and peripheral adrenergic nerve terminals, reduces the sensitivity of post synaptic alpha adrenergic receptors to catecholamines and also acts as a direct peripheral vasodilator.

The surgical approach of first choice is laparoscopic tumour removal. Laparoscopy in pheochromocytoma was thought to produce adverse hemodynamic effects as a result of the pneumo-peritoneum. But a recent study proved that the cardiac index or left ventricular work index were not affected significantly by the pneumoperitoneum created for laparoscopic adrenelectomy when compared to control group of patients undergoing open adrenelectomy. ${ }^{28}$ The rise in serum catecholamine concentration was found to be less with laparoscopy during tumour manipulation than with open adrenelectomy. ${ }^{17}$ Laparoscopic adrenelectomy has proved to be performed safely for excision of tumour less than 7 $\mathrm{cm}$ size. ${ }^{28}$ Being a less invasive procedure, this results in less post-operative pain and shorter hospital stay.

Funding: No funding sources

Conflict of interest: None declared

Ethical approval: Not required

\section{REFERENCES}

1. Lenders JW, Eisenhofer G, Mannelli M \& Pacak K. Pheochromocytoma. Lancet. 2005;366:665-75.

2. Wissler RN. Endocrine disorders. In: Chestnut DH, eds. Obstretic Anaesthesia. Principles and Practice. 2nd ed. New York: Mosby Year Book Inc.; 1999: 828-832.

3. Mastrogiannis DS, Whiteman VE, Mamopoulos M, Salamed WA. Acute endocrinopathies during pregnancy. Clin Obstet Gynaecol. 1994;37:78-92.
4. Harrington JL, Farley DR, van Heerden JA, Ramin KD. Adrenal tumours and pregnancy. World J Surg. 1999;23:182-6.

5. Grossman A, Pacak K, Sawaka A, Lenders JW, Harlander D, Peaston RT, Reznek R, Sisson J \& Eisenhofer G. Biochemical diagnosis \& localization of pheochromocytoma: can we reach a consensus? Ann NY Acad Sci. 2006;1073:332-47.

6. Lenders JW, Pacak K, Walther MM, Linehan WM, Mannelli M, Friburg P, Keiser HR, Goldstein DS, Eisenhofer G. Biochemical diagnosis of pheochromocytoma: which test is best? J Am Med Assoc. 2002;287:1427-34.

7. Shenker JG, Chowers I. Pheochromocytoma and pregnancy. Review of 89 cases. Obstet Gynaecol Surv. 1971;26:739-47.

8. Dean RE. Pheochromocytoma and pregnancy. Obstet Gynaecol. 1958;11:35-42.

9. Harper MA, Murnaghan GA, Kennedy L, Hadden DR, Atkinson AB. Pheochromocytoma in pregnancy. Five cases and a review of the literature. Br J Obstet Gynaecol. 1989;96:594-606.

10. Botchan A, Hauser R, Kupfermine M, Grisaru D, Peyser MR, Lessing JB. Pheochromocytoma in pregnancy: case report and review of the literature. Obstet Gynecol Surv. 1995 Apr;50(4):321-7.

11. Ahlawat SK, Jain S, Kumari S, Varma S, Sharma BK. Pheochromocytoma associated with preganancy: Case report and review of the literature. Obstet Gynaecol Surv. 1999;54:728-37.

12. Schenker JG, Granat M. Pheochromocytoma and pregnancy: an updated appraisal. Aus N Z J Obstet Gynaecol. 1982;22:1-10.

13. Oishi S, Sato T. Pheochromocytoma in pregnancy: a review of the Japanese literature. Endocrine J. 1994;41:219-25.

14. Bullough AS, Karadia S, Watters M. Pheochromocytoma: an unusual cause of hypertension in pregnancy. Anaesth. 2001;56:43-6.

15. Freier DT, Thompson NW. Pheochromocytoma and pregnancy: the epitome of high risk. Surg. 1993; 114:1148-52.

16. Almog B, Kupfermine MJ, Many A, Lessing JB. Pheochromocytoma in pregnancy: a case report and review of the literature. Acta Obstet Gynaecol Scand. 2000;79:709-11.

17. Inabnet $\mathrm{WB}$, Pitre $\mathrm{J}$, Bernard $\mathrm{D}$, Chapuis $\mathrm{Y}$. Comparison of the hemodynamic parameters of open and laparoscopic adrenalectomy for pheochromocytoma. World J Surg. 2000;24:574-8.

18. O'Riordan JA. Pheochromocytomas and anaesthesia. Int Anaesthesiol Clin. 1997;35:99-127.

19. Gagner M, Lacroix A, Prinz RA et al. Early experience with laparoscopic approach for adrenalectomy. Surg. 1993;114:1120-225.

20. Mannelli M, Bemporad D. Diagnosis and management of pheochromocytoma during pregnancy. J Endocrinol Invest. 2002;25:567-71. 
21. Burgess GE III. Alpha blockade and surgical intervention of pheochromocytoma in pregnancy. Obstet Gynaecol. 1979;53:266-70.

22. Cammarano WB, Gray AT, Rosen MA, Lim KH. Anaesthesia for combined caesarian section and extra adrenal pheochromocytoma resection: a case report and literature review. Int $\mathbf{J}$ Obstet Anaesth. 1997;6:112-7.

23. Stoelting RK, Dierdorf SF. Disease of the endocrine System. In: Stoelting RK, Dierdorf SF, eds. Anaesthesia and Coexisting Disease. 4th ed. New York: Churchill Livingstone Inc.; 2002: 430-434.

24. G. Edward Morgan Jr, Maged SM, Michael JM. Cisatracurium. In: 24. G. Edward Morgan Jr, Maged SM, Michael JM, eds. Clinical Anaesthesiology. 3rd ed. New York: McGraw-Hill; 2001: 192-193.

25. G. Edward Morgan Jr, Maged SM, Michael JM. Sevoflurane. In: G. Edward Morgan Jr, Maged SM,
Michael JM, eds. Clinical Anaesthesiology 3rd ed. New York: McGraw-Hill; 2001: 143-146.

26. James MF, Huddle KR, Owen KD, Van der Veen VW. Use of magnesium sulphate in the anaesthetic management of pheochromocytoma in pregnancy. Can J Anaesth. 1988;35:178-82.

27. Fernandez- Cruz L, Taura P, Saenz A, Bennarroch G \& Sabater L. Laparoscopic approach to pheochromocytoma: hemodynamic changes and catecholamine secretion. World J Surg. 1996;20:7628.

28. Janetschek G, Neumann HP. Laparoscopic surgery for pheochromocytoma. Urol Clin North Am. 2001;28:97-105.

DOI: 10.5455/2320-1770.ijrcog20140917

Cite this article as: Korichi N, Shaikh N, Mathew G, Alloub MA, Boursaly I, Scott N. Pheochromocytoma and pregnancy with abruptio placenta. Int J Reprod Contracept Obstet Gynecol 2014;3:772-6. 